\title{
On the number of independent sets in a tree
}

\author{
Hiu-Fai Law \\ Mathematical Institute \\ Oxford University \\ OX1 3LB, U.K. \\ lawh@maths.ox.ac.uk
}

Submitted: Feb 9, 2010; Accepted: Mar 15, 2010; Published: Mar 22, 2010

Mathematics Subject Classifications: 05C69, 05C05

\begin{abstract}
We show in a simple way that for any $k, m \in \mathbb{N}$, there exists a tree $T$ such that the number of independent sets of $T$ is congruent to $k$ modulo $m$. This resolves a conjecture of Wagner (Almost all trees have an even number of independent sets, Electron. J. Combin. 16 (2009), \# R93).
\end{abstract}

\section{The number of independent sets in a tree}

A set of vertices in a graph $G$ is called independent if the set induces no edges. We write $i(G)$ for the number of independent sets in $G ; i(G)$ is often known as the Fibonacci number, or in mathematical chemistry as the Merrifield-Simmons index or the $\sigma$-index. The study was initiated by Prodinger and Tichy in [4]. In particular, they showed that among trees of the same order, the maximum and minimum Fibonacci numbers are attained by the star and the path respectively. The name stems from the fact that the Fibonacci numbers of paths are the usual Fibonacci numbers. Indeed, as the empty set is independent, $i\left(P_{0}\right)=1, i\left(P_{1}\right)=2$ and $i\left(P_{n}\right)=i\left(P_{n-1}\right)+i\left(P_{n-2}\right)$ for $n \geqslant 2$.

The inverse question asks for a positive integer $k$, whether there exists a graph $G$ such that $i(G)=k$. Clearly there does as $i\left(K_{k-1}\right)=k$ (note that the empty set is independent). The question becomes more interesting if we restrict ourselves to certain classes of graphs. For the class of bipartite graphs, Linek [3] answered the question affirmatively. Here we are interested in the class of trees. For $k \in \mathbb{N}$, we say that $k$ is constructible if there exists a tree $T$ such that $i(T)=k$. For example, 1, 2, 3 are constructible (from the paths $P_{0}, P_{1}, P_{2}$ respectively) but 4 is not. In [3], Linek raised the following conjecture (see also $[2])$.

Conjecture 1 ([3]). There are only finitely many positive integers that are not constructible. 
An interesting paper of Wagner [5] looks at the number of independent sets modulo $m$. Wagner showed that the proportion of trees on $n$ vertices with the number of independent sets divisible by $m$ tends to 1 as $n$ tends to infinity. In the same paper, Wagner [5] proposed a weaker version of Conjecture 1. Let

$$
C(m)=\{i(T)(\bmod m): T \text { a tree }\} .
$$

Conjecture $2([5])$. For $m \in \mathbb{N}, C(m)=\mathbb{Z}_{m}$.

The aim of this paper is to prove Conjecture 2. In fact, we prove a stronger result. For a rooted tree $(T, r)$, let $i_{0}(T, r)$ denote the number of independent sets not covering the root. Let

$$
D(m)=\left\{\left(i_{0}(T, r), i(T)\right)(\bmod m):(T, r) \text { a rooted tree }\right\} .
$$

Theorem 3. For $m \in \mathbb{N}, D(m)=\mathbb{Z}_{m}^{2}$.

First we note a recursion between the Fibonacci number of a rooted tree and its subtrees. Suppose $r_{1}, r_{2}, \cdots, r_{j}$ are the neighbours of $r$, let $\left(T_{k}, r_{k}\right)$ be the subtree of $T$ rooted at $r_{k}$. Then we have $[2,5]$

$$
\begin{aligned}
i_{0}(T) & =\prod_{k=1}^{j} i\left(T_{k}\right) \\
i(T) & =\prod_{k=1}^{j} i\left(T_{k}\right)+\prod_{k=1}^{j} i_{0}\left(T_{k}, r_{k}\right)
\end{aligned}
$$

For rooted trees $\left(T_{1}, r_{1}\right), \cdots,\left(T_{j}, r_{j}\right)$, we write $\oplus_{k=1}^{j}\left(T_{k}, r_{k}\right)$ for the rooted tree obtained by adding a vertex $r$ joined to every root $r_{k}$. Let $\varphi(T, r)=\left(i_{0}(T, r), i(T)\right)$.

Let $\mu: \mathbb{Z}_{m}^{2} \longrightarrow \mathbb{Z}_{m}^{2}$ be the Fibonacci operator $(a, b) \mapsto(b, a+b)$. The sequence $\left(\mu^{k}(a, b): k \in \mathbb{N}\right)$ must contain repeated elements since $\mathbb{Z}_{m}^{2}$ is finite. But once it repeats, the sequence becomes periodic. Moreover, as $\mu$ is invertible with $\mu^{-1}(a, b)=(b-a, a)$, the sequence is periodic from the start. Denote by $[a, b]$ the orbit of $(a, b)$ under $\mu$.

In the following, we write $(a, b) \cdot(c, d)=(a c, b d)$ and $c \cdot(a, b)=(c a, c b)$.

Proposition 4. For $m \in \mathbb{N}$ with $C=C(m)$ and $D=D(m)$, we have

1. $(a, b) \in D \Rightarrow[a, b] \subset D$;

2. $[0,1] \subset D$;

3. $(a, b),(c, d) \in D \Rightarrow(a c, b d) \in D$; and

4. $c \in C,(a, b) \in D \Rightarrow(c a, c b) \in D$.

Proof. Let $\left(T_{1}, r_{1}\right),\left(T_{2}, r_{2}\right)$ be trees such that $\varphi\left(T_{1}, r_{1}\right) \equiv(a, b), \varphi\left(T_{2}, r_{2}\right) \equiv(c, d)(\bmod m)$. 
1. Consider $(T, r)$ obtained from $T_{1}$ by joining a new vertex $r$ to $r_{1}$. Then $\varphi(T, r)=$ $(b, b+a)$. Hence, $\mu(a, b) \in D$. Inductively adding a leaf to the root, the whole orbit $[a, b]$ lies in $D$.

2. Consider the paths: as $i_{0}\left(P_{0}\right)=i\left(P_{0}\right)=1$, we have $\left.[0,1]=1,1\right] \subset D$.

3. $\varphi\left(\left(T_{1}, r_{1}\right) \oplus\left(T_{2}, r_{2}\right), r\right)=(b d, b d+a c)$. By $(1),[b d, b d+a c] \subset D$. Note that $(a c, b d)=$ $\mu^{-1}(b d, b d+a c)$.

4. If $c \in C$, then $(x, c) \in D$ for some $x$. As $(0,1) \in D$, it follows that $(x, c) \cdot(0,1)=$ $(0, c) \in D$. Then $(c, c)=\mu(0, c) \in D$. By $(3),(c, c) \cdot(a, b)=(a c, b c) \in D$.

Proposition 5. For all $x \in \mathbb{Z}_{m},(1, x)$ and $(x, 1)$ are in $D(m)$.

Proof. By Proposition 4, $[0,1] \subset D$. Moreover, the Fibonacci sequence looks like

$$
\cdots, 2,-1,1,0,1,1,2, \cdots
$$

Thus, $-1 \in C$ and $(1,1),(-1,1),(1,2),(2,-1) \in D$. Moreover, since $-1 \in C,-1$. $(-1,1)=(1,-1) \in D$.

Suppose $(1, a),(a, 1) \in D$. Applying Proposition 4, we have that each of the following is in $D$.

$$
\begin{aligned}
& (1, a) \stackrel{\mu^{-1}}{\Rightarrow}(a-1,1) \stackrel{\cdot(1,-1)}{\Rightarrow}(a-1,-1) \stackrel{\mu}{\Rightarrow}(-1, a-2) \stackrel{\cdot(-1,1)}{\Rightarrow}(1, a-2) \\
& (a, 1) \stackrel{\mu}{\Rightarrow}(1, a+1) \stackrel{\cdot(-1,1)}{\Rightarrow}(-1, a+1) \stackrel{\mu^{-1}}{\Rightarrow}(a+2,-1) \stackrel{\cdot(1,-1)}{\Rightarrow}(a+2,1) .
\end{aligned}
$$

Applying the argument repeatedly to $(1,1),(1,2)$ and $(2,1)$, we have that $\{(1,1-2 b)$, $\left.(1,2-2 b),(2+2 b, 1),(1+2 b, 1): b \in \mathbb{Z}_{m}\right\}=\left\{(1, x),(x, 1): x \in \mathbb{Z}_{m}\right\} \subset D$.

Proof of Theorem 3. For $(x, y) \in \mathbb{Z}_{m}^{2}$, take $(x, 1)$ and $(1, y)$ in $D$ and multiply them.

We remark that the trees in our construction have maximum degree 3 , so that for any integer $m>0,\{i(T)(\bmod m): T$ a tree, $\Delta(T) \leqslant 3\}=\mathbb{Z}_{m}$. This is in contrast to the result in [1] that the Fibonacci numbers (of the paths) form a complete system of residues if and only if $m=t \cdot 5^{k}, t=1,2,4,6,7,14,3^{j}$ where $k \geqslant 0, j \geqslant 1$.

\section{The number of matchings in a tree}

In this section, we turn to the number of matchings in a graph. This is also known as the Hosoya index, or the $Z$-index in mathematical chemistry. For a rooted tree $T$, let $Z(T)$ be the number of matchings and $Z_{0}(T)$ be those not covering the root. In [5], Wagner also mentioned that for any $m \in \mathbb{N}$, the proportion of trees on $n$ vertices with $Z(T)$ a multiple of $m$ tends to 1 as $n$ tends to infinity.

The inverse problem in the family of trees is easy because $Z\left(K_{1, k-1}\right)=k$, [2]. Let $B(m)=\left\{\left(Z_{0}(T), Z(T)\right)(\bmod m): T\right.$ a rooted tree $\}$. Note that we consider the empty set as a matching as well. Applying the previous technique, we will show the following. 
Theorem 6. For $m \in \mathbb{N}, B(m)=\mathbb{Z}_{m}^{2}$.

There are formulae for matchings analogous to (1) and (2), see [5]. However, here we find it more convenient to consider joining two rooted trees $\left(T_{1}, r_{1}\right),\left(T_{2}, r_{2}\right)$ by adding the edge $r_{1} r_{2}$ to form $T$ rooted at $r_{1}$. Then

$$
\begin{aligned}
Z_{0}\left(T, r_{1}\right) & =Z_{0}\left(T_{1}, r_{1}\right) Z\left(T_{2}\right) \\
Z(T) & =Z\left(T_{1}\right) Z\left(T_{2}\right)+Z_{0}\left(T_{1}, r_{1}\right) Z_{0}\left(T_{2}, r_{2}\right)
\end{aligned}
$$

Let $(a, b) \odot(c, d)=(a d, a c+b d)$.

Proposition 7. For $m \in \mathbb{N}, B=B(m)$, we have

1. $(a, b),(c, d) \in B \Rightarrow(a d, a c+b d) \in B$

2. $(c, d) \in B \Rightarrow[c, d] \subset B$;

3. $[0,1] \subset B$; and

4. $(c, d) \in B \Rightarrow(d, c) \in B$.

Proof. 1. Join the two trees corresponding to $(a, b),(c, d)$ at the roots and root it at the first one. Then $(a, b) \odot(c, d)=(a d, a c+b d) \in B$.

2. By 1 , if $(c, d) \in B$, then $(1,1) \odot(c, d)=(d, c+d) \in B$. (Note that attaching a new vertex to the root has exactly the same effect as in the proof of Proposition 4.)

3. Consider the paths: as $Z\left(P_{0}\right)=1$ and $Z\left(P_{1}\right)=1$, we have $(1,1) \in B$ so that $[1,1]=[0,1] \subset B$.

4. As $(1,0) \in[0,1] \subset B$, we have $(1,0) \odot(c, d)=(d, c) \in B$.

Proof of Theorem 6. We first show that $(1, a) \in B$ for all $a \in \mathbb{Z}_{m}$. As $[0,1] \subset B,(-1,1)=$ $\mu^{-2}(0,1)$ and $(1,1)=\mu(0,1)$ are in $B$. Suppose $(1, a) \in B$, then by Proposition 7 , $(1, a) \odot(-1,1)=(1, a-1) \in B$. Repeating the operation with $(-1,1)$, we have $(1, a) \in B$ for all $a$. Moreover, $(0,1) \odot(1, a)=(0, a)$ is in $B$ for all $a$ as well.

Suppose for a fixed $1 \leqslant k<m$, we have $\{(i, a): 0 \leqslant i \leqslant k-1, \forall a\} \subset B$. In particular, $(i, k) \in B$ for all $0 \leqslant i \leqslant k-1$. Now as $(1,-1) \odot(i, k)=(k, i-k)$, applying Proposition 7 , we get that $(i-k, k) \in B$. Hence, $\{(i-a k, k): 0 \leqslant i \leqslant k-1, \forall a\} \subset B$. This shows that for all $a,(a, k) \in B$ which, by Proposition 7 , implies that $(k, a) \in B$. Repeating the argument by increasing $k$, we conclude that $B=\mathbb{Z}_{m}^{2}$. 


\section{References}

[1] S.A. Burr, On moduli for which the Fibonacci sequence contains a complete system of residues, Fibonacci Quart. 9 (1971), 497-504.

[2] X. Li, Z. Li, and L. Wang, The inverse problems for some topological indices in combinatorial chemistry, J. Comput. Biol. 10 (2003), 47-55.

[3] V. Linek, Bipartite graphs can have any number of independent sets, Discrete Math. 76 (1989), 131-136.

[4] H. Prodinger and R.F. Tichy, Fibonacci numbers of graphs, Fibonacci Quart. 20 (1982), $16-21$.

[5] S.G. Wagner, Almost all trees have an even number of independent sets, Electron. J. Combin. 16 (2009), \# R93. 